\title{
Application of Continuous Quality Improvement in Manual Cleaning of Fine Hollow Device
}

\author{
Zeng Xiuyue ${ }^{1}$, Zhang Lian ${ }^{1}$, Chen Chunyan ${ }^{1, ~ *, ~ Y u ~ H a o h u i ~}{ }^{2}$ \\ ${ }^{1}$ Central Sterile Supply Department, The First Affiliated Hospital of Jinan University, Guangzhou, China \\ ${ }^{2}$ Department of Hospital Infection Control, The First Affiliated Hospital of Jinan University, Guangzhou, China
}

Email address:

zmonna $126 . c o m$ (Zeng Xiuyue), zlscdz@163.com (Zhang Lian), 1553430760@qq.com (Chen Chunyan)

${ }^{*}$ Corresponding author

To cite this article:

Zeng Xiuyue, Zhang Lian, Chen Chunyan, Yu Haohui. Application of Continuous Quality Improvement in Manual Cleaning of Fine Hollow Device. American Journal of Nursing Science. Vol. 9, No. 1, 2020, pp. 43-46. doi: 10.11648/j.ajns.20200901.17

Received: January 14, 2020; Accepted: January 31, 2020; Published: February 11, 2020

\begin{abstract}
Objectives: The study aims to explore the effect of continuous quality improvement on manual cleaning quality of fine hollow device in hospital central sterile supply department. In terms of methods, it mainly optimize the manual cleaning process of fine hollow device as follow: Seperated cleaning basket is employed with $3 \%$ hydrogen proxide solition to clean the blood stains and organic matters in fine hollow device. And then, $90^{\circ} \mathrm{C}$ pure water is used to soak the fine hollow device to dissolve and clean the solidly attached bone wax in the inner side of absorption tube. And the study primarily compares the difference between the data about optimizing the manual cleaning process of fine hollow device through continuous quality improvement, the blood (stain), tissue residue, foreign body residue, bone wax from August to September in 2019, and those from October to November in 2019. Results: The quality of fine hollow device manual cleaning in reference group are improved significantly, with outstanding differences in control group $\left(\mathrm{X}^{2}=59.36, \mathrm{P}=0.000\right)$, indicating the effectiveness of the continuous improvement measures. Conclusion: The continuous quality improvement was applied to the manual cleaning of fine hollow device, and the cleaning effect was satisfactory. The removal rate of blood (stain), tissue, foreign body and bone wax of fine hollow device was improved.
\end{abstract}

Keywords: Continuous Quality Improvement, Fine Hollow Device, Manual Cleaning, Central Sterile Supply Department, Cleaning Effect

\section{Introduction}

Fine hollow device (diameter $<2 \mathrm{~mm}$ ) are widely used in minimally invasive surgery [1]. Because of its complex structure, many grooves, high precision and slender pipe diameter, it is difficult to clean the equipment, and the rate of washing back is high. However, the presence of blood stains, tissues, foreign bodies and bone wax in the cavity for a long time can form biofilm, which can not only easily corrode the equipment, but also affect the sterilization effect and increase the risk of cross-infection [2-3]. Therefore, improving the cleaning, disinfection and sterilization quality of such fine hollow device has become the operation difficulty of hospital disinfection supply center, and it is also the management focus of controlling nosocomial infection. In order to reduce the risk of infection caused by fine hollow device, the hospital disinfection supply center specification (WS310-2016) emphasizes in particular that fine and complex instruments are suitable for manual cleaning [4]. Brain, otolaryngology and some orthopaedic hollow device belong to fine hollow device, because they often adhere to a large number of blood stains and organic matter, and the most difficult to remove bone wax, which brings great challenges to manual cleaning. Our hospital central sterile supply department (CSSD) for continuous quality improvement management of manual cleaning of fine hollow device in 2019, achieved remarkable results, which are reported as follows.

\section{Data and Methods}

\subsection{General Data}

Retrospective analysis of the cleaning quality data of 
CSSD fine hollow device manual cleaning in our hospital in August-September 2019 control group; analysis of October-November 2019 improvement The cleaning quality data after the manual cleaning method of the rear fine lumen instruments are set as Watch team. Yes Method of manual cleaning of fine lumen instruments Two sets of data comparison, analysis Fine lumen instruments Blood stain, tissue residue, foreign body residue, bone wax residue.

\subsection{Method}

The control group carried on the manual cleaning to the fine lumen instrument according to the conventional way, the observation group carried on the operation according to the improved fine lumen instrument manual cleaning method, the concrete is as follows.

\subsubsection{Observation Group}

$3 \%$ hydrogen peroxide solution with separate cleaning basket Decomposition of organic matter and $90^{\circ} \mathrm{C}$ Pure soaking Dissolve bone wax. The details are as follows: 1 Initial flushing and scrubbing under flowing water Fine lumen instruments Visible to the naked eye Blood stains, tissues, foreign bodies, bone wax; injection of enzyme solution into tube cavity and immersion in multi-enzyme solution for $10 \mathrm{~min}$; brushing of tube cavity with suitable brush; 3. Ultrasonic cleaning of enzyme solution for $3 \mathrm{~min}$ The inside and outside of the lumen and the concave and convex areas are thoroughly hand-brushed, and the inner wall of the lumen is repeatedly washed with a high pressure water gun to remove the lumen Internal and external residues 4. Immerse in $3 \%$ hydrogen peroxide solution Break down, will lumen Internal injection Soak the solution for 1-2 min below the surface, then remove the solution Rinse the lumen of the solution until No white bubbles; use high pressure water gun to wash the inner wall of the lumen repeatedly, fully rinse the inside and outside of the lumen; this step is mainly to remove blood (stain) trace, organic residue; 5 use $90^{\circ} \mathrm{C}$ hot pure water to soak, dissolve bone wax and disinfect instrument, inject into the lumen Full of hot water Under liquid level Soak for $10 \mathrm{~min}$; observe the oil stains on the hot pure water surface at $90^{\circ} \mathrm{C}$, wash the inner wall of the lumen repeatedly with a suitable brush of the appropriate size, and soak and sterilize again at $90^{\circ} \mathrm{C}$ hot pure water surface until there is no oil stain on the hot pure water surface at $90^{\circ} \mathrm{C}$; this step is mainly to remove the residue of bone wax; 6 . Fine lumen instruments Place in a drying cabinet with a tilt of $30^{\circ} \mathrm{C}$ and a drying temperature of $70-90^{\circ} \mathrm{C} ; 7$. Use a visual + light source magnifying glass before checking the packaging; and Use white gauze to cover one end of the lumen, air gun and then the other end of the repeated blow, check for foreign bodies and tissue residues.

\subsubsection{Control Group}

The traditional cleaning method was used to carry out manual cleaning of fine lumen instruments. the manual cleaning method is the same as the observation group' s 1236, the difference is that after full rinsing, the washed fine lumen instruments are put into the boiling device to boil at $90^{\circ} \mathrm{C}$ for 1 $\min$.

\subsubsection{Cleaning Methods Training and Assessment}

Training and assessment of cleaning staff by decontamination area leader [5].

\subsection{Observation Indicators}

Use visual + light source magnifying glass [6]+ Air gun blowing Gas is the most commonly used method of cleaning quality inspection of lumen instruments in clinic, the equipment after cleaning should be clean and clean, no blood stains, stains, tissues, bone wax and other residues, and record the results of the inspection.

\subsection{Statistical Analysis}

SPSS 19.0 software was used for statistical analysis. The measurement data are compared by $\mathrm{t}$ test or variance analysis, and the counting data are compared by $\mathrm{X}^{2}$ test, $\mathrm{P}<0.05$ indicated that the difference was statistically significant.

\section{Results}

\subsection{Before and After Improvement of Manual Cleaning Process in Fine Lumen Two Groups Comparison of Cleaning Quality}

Improvement of manual cleaning of fine lumen instruments after implementation of the observation group Cleaning quality improved, two groups Data Significant differences $\left(\mathrm{X}^{2}=59.36, \quad \mathrm{P}=0.000\right)$, indicating the effectiveness of the continuous improvement measures. See table 1 .

Table 1. Before and after improvement of manual cleaning process in fine lumen Two groups Comparison of cleaning quality.

\begin{tabular}{lllllll}
\hline Group & blood stain & Bone wax & Organization & Foreign bodies & Total number of cases & $\mathbf{X}^{2}$ \\
\hline Control group & 52 & 23 & 12 & 3 & 728 & \\
Observation group & 18 & 2 & 4 & 1 & 931 & 5.000 \\
\hline
\end{tabular}

\subsection{Before and After Improvement of Manual Cleaning Process in Fine Lumen Two Groups Comparison of Blood Removal}

Improving the manual cleaning process of fine lumen instruments higher clearance rate of blood stains Control group. Two sets of data Significant differences $\left(X^{2}=27.434\right.$, $\mathrm{P}=0.000$ ), indicating the effectiveness of the continuous improvement measures. See table 2. 
Table 2. Before and after improvement of manual cleaning process in fine lumen Two groups Comparison of blood removal.

\begin{tabular}{lllll}
\hline Group & blood stain & No blood stains & $\mathbf{X}^{\mathbf{2}}$ & $\boldsymbol{P}$ \\
\hline Control group & 52 & 676 & 27.4 & \multirow{2}{*}{0.000} \\
Observation group & 18 & 913 & 34 & \\
\hline
\end{tabular}

\subsection{Before and After the Improvement of the Manual Cleaning Process in the Fine Lumen Two Groups Comparison of Clearance of Bone Wax}

After improving the manual cleaning process of fine lumen instruments, the observation group Bone wax clearance rate higher Control group. Two sets of data Significant differences $\left(\mathrm{X}^{2}=23.865, \mathrm{P}=0.000\right)$, indicating the effectiveness of the continuous improvement measures. See table 3.

Table 3. Before and after the improvement of the manual cleaning process in the fine lumen Two groups Comparison of clearance of bone wax.

\begin{tabular}{lllll}
\hline Group & Bone wax & No bone wax & $\mathbf{X}^{2}$ & $\boldsymbol{P}$ \\
\hline Control group & 23 & 705 & 23.8 & \multirow{2}{*}{0.000} \\
Observation group & 2 & 929 & 65 & \\
\hline
\end{tabular}

\subsection{Before and After the Improvement of the Manual Cleaning Process in the Fine Lumen Two Groups Comparison of Organizational Clearance}

After improving the manual cleaning process of fine lumen instruments, the observation group Organization clearance rate higher than Control group. Two sets of data Differences $\left(\mathrm{X}^{2}=6.535, \mathrm{P}=0.012\right)$, indicating the effectiveness of the continuous improvement measures. See table 4.

Table 4. Before and after the improvement of the manual cleaning process in the fine lumen Two groups Comparison of organizational clearance.

\begin{tabular}{lllll}
\hline Group & Organization & No Organization & $\mathbf{X}^{\mathbf{2}}$ & $\boldsymbol{P}$ \\
\hline Control group & 12 & 716 & 6.535 & 0.012 \\
Observation group & 4 & 927 & & \\
\hline
\end{tabular}

3.5. Before and After the Improvement of the Manual Cleaning Process in the Fine Cavity Two Groups Comparison of Foreign Body Removal

After improving the manual cleaning process of fine lumen instruments, the observation group Foreign body clearance and Control group No change. Two sets of data No difference $\left(\mathrm{X}^{2}=0.564, \mathrm{P}=0.453\right)$. See table 5 .

Table 5. Before and after the improvement of the manual cleaning process in the fine cavity Two groups Comparison of foreign body removal.

\begin{tabular}{lllll}
\hline Group & $\begin{array}{l}\text { Foreign } \\
\text { bodies }\end{array}$ & $\begin{array}{l}\text { No foreign } \\
\text { bodies }\end{array}$ & $\mathbf{X}^{2}$ & $\boldsymbol{P}$ \\
\hline $\begin{array}{l}\text { Control group } \\
\text { Observation group }\end{array}$ & 3 & 725 & 0.564 & 0.453 \\
\hline
\end{tabular}

\section{Discussion}

\subsection{Prevent Leakage and Contamination of Fine Lumen Instruments}

Normally, a set of brain and facial lumen instruments has
5-9 tubes with different diameters. During manual cleaning, it is easy for the cleaners to mix the unwashed lumen instruments into the cleaned lumen instruments and contaminate other instruments during boiling disinfection and cleaning quality inspection. During the cleaning process, there are few leakages of the lumen instruments after the basket is loaded. Put an end to the phenomenon that other instruments are contaminated by leakage washing of lumen instruments.

\subsection{Use 3\% Hydrogen Peroxide Solution to Effectively Remove Dried Blood Stains and Organic Matter from the Inner Wall of the Lumen Apparatus}

Fine lumen instruments have the characteristics of small or long pipe diameter, after cleaning, it is easy to leave blood stains, tissue, protein and other organic matter in the inner wall of the pipe diameter after cleaning, and the difficulty coefficient of thoroughly cleaning the residual organic matter in the lumen is high [7]. The $3 \%$ hydrogen peroxide solution is rich in many kinds of protein decomposing enzymes, which can effectively remove the residual blood stains, tissues, proteins and other organic matter in the instrument. After the initial washing, washing, enzyme soaking and washing, ultrasonic washing and water gun washing, the tube cavity is washed repeatedly with $3 \%$ hydrogen peroxide solution and soaked for 1-2 min, which can more thoroughly dissolve the residual blood stains, tissues and proteins in the inner wall of the pipe diameter. This result is consistent with the results of Li Liang [8].

\subsection{Use $90^{\circ} \mathrm{CHeat}$ Soak in Pure Water to Effectively Remove the Bone Wax Firmly Attached to the Inner Wall of the Suction Tube}

The main components of bone wax are beeswax, vegetable oil, salicylic acid [9]. It is widely used in orthopedics [10], brain department [11], five faculties [12] surgery for bone hemostasis [13]. The dosage can be used to achieve hemostasis, and the surrounding excess bone wax is removed with lumen instruments. When the bone wax is sucked out by the instrument of the lumen, some parts are firmly adhered to the inner wall of the lumen, which cannot be removed by using the general cleaning method. According to its preparation process and confirmed by experiments, the residual bone wax in the instrument of the lumen is completely melted and precipitated after soaking in hot pure water at $90^{\circ} \mathrm{C}$ for $10 \mathrm{~min}$.

\section{Conclusions}

The CSSD of our hospital is loaded by separate cleaning basket, $3 \%$ hydrogen peroxide solution cleaning and $90^{\circ} \mathrm{C}$ heat measures such as pure water immersion improve the manual cleaning quality of fine lumen instruments, improve the removal rate of blood stains, tissues, proteins and other organic matter in the inner wall of the pipe diameter, ensure the cleaning quality of fine lumen instruments, and also have an important role in promoting the control of nosocomial infection. 


\section{References}

[1] Zhang Junying, Wang Ke, Zhou Pu. Effect of different pre-cleaning methods on lumen instruments [J]. Chinese Journal of Nosocomial Infection, 2016, 26 (19): 4551-4553.

[2] Zhou Xue, Wei Jingrong, Ren Xiaomei. Improvement and effect observation of cleaning quality control method of surgical instruments in lumen [J]. Journal of Surgery, 2014, 23 (4): 357-359.

[3] Liu Yanling, Wu Shuhong, Wang Zhengxu. Study on Cleaning and Disinfecting Methods of Tube and Cavity Multiplexing Instruments [J]. Chinese Journal of Nosocomial Infection, 2014, 24 (15) 3876-3878.

[4] National Health and Family Planning Commission. Health Industry Standards of the People's Republic of China [S]. Hospital Disinfection Supply Center-Part 2: Technical Specification for Cleaning, Disinfection and Sterilization, 2016: 12.

[5] Zhao Huijie, Wang Lihong, Zhang Jingli. Measures to improve the cleaning effect of lumen instruments $[\mathrm{J}]$. Chinese Journal of Disinfection, 2018, 35 (1): 50-54.

[6] National Health and Family Planning Commission. Health Industry Standards of the People's Republic of China [S]. Hospital Disinfection Supply Center-Part 3: Monitoring Standards for Cleaning and Disinfection and Sterilization Effects, 2016: 12.

[7] Zhou Xiaoyu. Application effect of quality control circle activity in the improvement of cleaning quality process of lumen instruments $[\mathrm{J}]$. General Nursing, 2018, 16 (23): 2923-2925.

[8] Li Liang. Analysis of Different Cleaning Methods of Disinfection Supply Center in Brain Drawer's Cleaning Effect [J]. Medical Theory and Practice, 2019, 10 (32): 1605-1606.

[9] Pang Lin. Improvement in prescription of bone wax [J]. Modern Chinese Applied Pharmacy, 2000, 17 (S 1): 102.

[10] Wanfu Silver, Guo Wanshou, Zhang Qidong, Cheng Liming. Efficacy and safety evaluation of osteowax in hemostasis after total knee arthroplasty [J]. Chinese Journal of Orthopaedic Surgery, 2015, 23 (17): 1576-1580.

[11] Jiang Weilian, Xie Lixia. Application of bone wax in titanium plate fixation in craniocerebral surgery [J]. Journal of Nursing Studies, 2011, 26 (06): 500.

[12] Liu Tiancong, Yu Xiaofeng, Gu Zhaowei, Bai Weiliang, Wang Zhenhai, Cao Zhiwei. Causes and management of frontal sinusitis after transfrontal craniotomy [J]. Journal of Clinical Otolaryngology and Head and Neck Surgery, 2018, 32 (03): 187-189.

[13] Yin Rongzhong, Dao Hongying. Preparation and Discussion of Bone Wax in Banna Area [J]. Chinese Journal of Ethnic Medicine, 2010, 16 (11): 33-34. 